\title{
Life course approach to prevention and control of non-communicable diseases
}

\section{A successful approach to reducing the burden of non-communicable disease requires action at all stages of life, argue Bente Mikkelsen and colleagues}

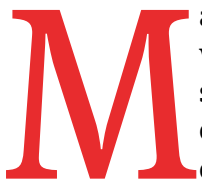

any of the health problems we encounter in adulthood stem from our experiences early in life-in some cases, even from before we are born. ${ }^{1-9}$ The major non-communicable diseases (NCDs) (diabetes, cardiovascular diseases, cancer, chronic respiratory diseases, and mental disorders) are often associated with older age groups, but the evidence suggests that they affect people of all ages. Fifteen million deaths attributed to NCDs occur between the ages of 30 and 69 years and people from all age groups are vulnerable to the risk factors that contribute to NCDs. ${ }^{10}$

NCD prevention is most effective when it targets a problem at its roots. Taking early, appropriate, timely, and collective action $^{1}$ is important if we are to reduce premature mortality related to NCDs by a third by 2030-a sustainable development goal that has been affirmed through political declarations by heads of states and governments. A life course approach is an inclusive approach that considers the needs of all age groups and addresses NCD prevention and control in its earliest stages and is recommended in the World Health Organization's global action plan for prevention and control of NCDs. ${ }^{11}$

\section{KEY MESSAGES}

- Through political declarations, heads of states and governments have committed to reduce premature deaths by $30 \%$ before 2030 .

- A set of cost effective and affordable policy options (best buys) exist to tackle NCDs

- Interventions to reduce risk from non-communicable diseases should be applied through the lifecourse.

- Actions targeted at one life stage often also influence health behaviour and outcomes at other stages

- The life course approach can help determine when and how to influence the social determinants of health
The life course approach is underpinned by evidence from a wide range of disciplines showing how NCDs are influenced by early life factors. ${ }^{12}{ }^{13}$ It provides a comprehensive and sustainable framework for identifying key settings for interventions, knowledge translation, and a systems thinking approach. ${ }^{14}$ WHO uses the theory as a basis for many of its strategies and recommendations. ${ }^{15-915-19}$

In this article we outline how a life course approach can be used to inform implementation of NCD prevention and control. We consider life stages from preconception to old age, and ways in which transitions in stage of life present opportunities for promoting health. We draw primarily on work from the European Region of WHO, which made life course approaches a major part of Health $2020^{19}$ and the regional framework for prevention and control of NCDs. ${ }^{20}$ Here we will cover recommendations and considerations within the stages of preconception through the periods of childhood, adolescence, and old age. Across the life course, WHO has recommendations for the prevention of NCDs that will affect all age groups, ${ }^{21}$ and these are discussed in more detail in the appendix on bmj.com. To reduce NCDs, it is also crucial to tackle the upstream determinants of health. Health policies must include the unique challenges posed by poverty, such as poor housing conditions and lack of social security, which are associated with poor health outcomes. Public policy should be developed for a coordinated response to the structural and social determinants of health and exposure to unhealthy environments.

Although we focus on a national level, the principles and themes are applicable to cities, schools, workplaces, and other local settings.

\section{Stages of the life course}

The main risk factors for non-communicable diseases are tobacco use, harmful use of alcohol, lack of physical activity, unhealthy diet, and air pollution. Throughout all stages of life, there are ways in which these risk factors may be targeted to help prevent the development of NCDs and mental health disorders later in life.

Priority interventions include both population and individual level activities. Population level interventions that have been identified as good value for money include regulations and taxations on tobacco and alcohol, promotion of healthy foods through transformed marketing policies, including improved packaging of commercial products, and revised fiscal strategies. Commercial products can be reformulated to reduce salt, saturated fats, and sugar consumption, and health literacy can be promoted by educating at-risk populations, so that they recognise the importance of healthy options. Active living and mobility can also be promoted. ${ }^{4}$ Air pollution is responsible for millions of deaths each year, thus, clean air is an essential component of NCDs prevention. ${ }^{4}$ Some of these interventions will be more relevant and efficient at certain stages of the life course, but most of them will affect people at all ages. The same is true for the interventions aimed at individuals, such as cardiometabolic risk assessment and management, early detection and effective treatment of major NCDs, and vaccination and relevant communicable disease control.

The life course approach is an intuitive way to conceptualise NCD prevention and control. It provides a comprehensive and sustainable framework to introduce key interventions for improved health literacy and knowledge translation. Additionally, it provides an avenue for adopting a complex systems model of public health. ${ }^{22}$

\section{Preconception and prenatal care}

The preconception period refers to a woman's health before she becomes pregnant, and the prenatal period refers to the time from conception up to the child's birth. Evidence is growing that a woman's nutritional status during these periods may influence her offspring's health and susceptibility to NCDs later in life. ${ }^{7}$

WHO recommends that before and during pregnancy, promoting healthy nutrition 
and regular physical activity can prevent hypertension and gestational diabetes. ${ }^{78}$ Unborn children are adversely affected by harmful exposures such as air pollution, tobacco use, and maternal consumption of alcohol. $^{523}$

Focused public health policies and primary healthcare services promote access to quality services during the preconception phase. Essential interventions include monitoring weight and counselling on nutrition and exercise. These are essential components of primary healthcare, and linkages to maternal health systems can facilitate early access to prenatal care. ${ }^{7}$ During pregnancy, health professionals should continue weight management and support physical activity, to improve the health of the mother and her child. ${ }^{8}$

Pregnancy presents an opportunity for family centred health promotion. Household members should be advised to eliminate tobacco use, to reduce alcohol consumption, and to eliminate air pollution within the home.

\section{Infancy}

Infancy is an extremely important stage for the prevention of NCDs later in life. ${ }^{12}$ A WHO systematic review of the literature concludes that a person's propensity to develop NCDs and obesity may be influenced during fetal development and infancy, and these factors may in part explain the observed correlation between health inequalities and NCDs. ${ }^{24}$

Exclusive breastfeeding prevents NCDs and helps ensure healthy newborn development, as outlined in a WHO systematic review. ${ }^{7}$ Public policy has a key role in promoting breastfeeding, ${ }^{6}$ including through national labour policies supporting universal paid maternity leave and requiring workplaces to provide suitable accommodation for breastfeeding mothers, such as appropriate breaks and facilities. ${ }^{7}$ National efforts are also needed to restrict the inappropriate marketing of products that compete with breastfeeding. ${ }^{6}$

The health system should promote breastfeeding, as outlined in the baby friendly hospital initiative, as well as monitoring the child's growth and the micronutrient status of both mother and newborn ${ }^{7}$ and providing behaviour change support related to physical activity, diet, or substance use, where necessary.

Infancy is also a key time for providing vaccinations, including hepatitis $B$ vaccinations to protect against liver cancer. $^{2025}$
A crucial consideration here is the child's environment-in the home, in day care centres, or in nursery facilities. In structuring these environments, a primary aim is to mitigate the infant's exposure to harmful influences such as secondary tobacco smoke, air pollution, and other environmental toxins.

\section{Childhood}

Children are exposed to multiple settings where new NCD related risks may be encountered. Kindergartens, schools, and preschools are perhaps the most important, partly because most children experience them and partly because they are a good setting for health promotion activities.

Physical activity and a healthy diet in childhood are prerequisites for healthy development. It is therefore important to structure children's environments in ways that result in sufficiently high physical activity levels and low levels of consumption of energy dense, nutrient poor foods. ${ }^{15}$ Health promoting schools, nurseries, or kindergartens can design their environments and practices in ways that steer children towards greater fruit and vegetable consumption and increased physical activity. ${ }^{20}$ Additionally, it is important to provide opportunities for children to actively travel to school such as safe footpaths and cycle lanes. ${ }^{26}$

Policy makers should also consider creating national standards for the food and drinks available in schools, placing restrictions on the marketing of unhealthy foods (including social marketing), mandating smoke-free childcare facilities, or monitoring the air in schools and public recreational settings to ensure that they meet WHO indoor air quality guidelines. ${ }^{20}$

Schools are also a good place to monitor risk factors for NCDs, and the data can be used to guide national prevention policies.. For example, the WHO European Childhood Obesity Surveillance Initiative measures trends in overweight and obesity among primary schoolchildren in more than 40 European countries, ensuring the availability of high quality data to inform policy and practice, and to respond to the problem of childhood overweight and obesity. $^{27}$

\section{Adolescence}

Adolescence, defined as the transitional phase between childhood and adulthood, is a time when young people begin developing habits that will carry over into adulthood and have large implications for their NCD risk. At this age, important settings for health promotion include healthy school environments (described above), home environments, the neighbourhood on the journey to and from school, and afterschool clubs and sports clubs.

Adolescents are vulnerable to marketing of harmful substances such as alcohol and tobacco. ${ }^{28}$ Countries must strengthen implementation of the WHO Framework Convention on Tobacco Control and tackle emerging risks such as electronic nicotine delivery systems. ${ }^{23}$ Mental health also becomes increasingly important during adolescence, ${ }^{29}$ and prevention of bullying and provision of school based counselling are vital. ${ }^{30}$

Healthy behaviours initiated in childhood, such as physical activity and healthy nutrition, should be maintained during adolescence. ${ }^{683132}$ A priority for policy should be to develop a coordinated response to the structural and social determinants of adolescent obesity, ${ }^{31}$ food insecurity, ${ }^{31}$ poor access to healthful food, and exposure to unhealthy environments. Although it is important at all stages of life, ${ }^{16}$ health literacy is highly valuable in adolescence as young people begin to make their own decisions related to their health. Finally, provision of HPV vaccinations to adolescent girls has been shown to be cost effective as part of a comprehensive approach to cervical cancer prevention. ${ }^{4}$

To inform policy action, it is important to collect information about individual risk factor behaviours, as well as information about the implementation and effectiveness of health promotion interventions. ${ }^{8}$ The Health Behaviour in School Aged Children ${ }^{31}$ initiative is a useful guide to measuring NCD risk factors during these important years.

\section{Adulthood}

The workplace is an important setting for health promotion during adulthood. ${ }^{20}$ Interventions that have been shown to be effective include promoting healthy food options in canteens and by offering nutrition education and counselling. ${ }^{4}$ Workplace policies restricting alcohol and tobacco use are important for the health of all employees. Providing opportunities and incentives for physical activity (including active transport) can promote mental health, prevent and rehabilitate musculoskeletal disorders, and improve heart health. ${ }^{33}$

Policies to improve health behaviours only through workplace settings will exclude groups most likely to have NCDs, such as unemployed people. To ensure that interventions do not widen inequalities 
further, it is important to integrate similar health promoting interventions in other accessible settings, including community centres, churches, healthcare settings, rehabilitation centres, and recreational facilities.

Among the many opportunities for improving NCD related surveillance during adulthood, monitoring alcohol and tobacco use is particularly important for purposes of targeting interventions, monitoring progress, and advocacy. ${ }^{34}$ Patterns related to tobacco and alcohol use, physical activity, and nutrition among adults should be measured alongside socioeconomic, demographic, or geographical variables. Population based surveillance can strengthen targeted cost effective approaches to NCD prevention and early intervention.

Governments can introduce various policies to reduce NCDs in adulthood. Fiscal policies, for instance, could tax unhealthy products such as tobacco, alcohol, or sugary drinks, and fund schemes that subsidise fruit and vegetables. ${ }^{35}$ Policies that promote mental health, such as strengthening leadership and governance and providing comprehensive mental health and social care services, are also beneficial at a time when people may face unsatisfying careers, unemployment, financial stressors, low social engagement, divorce, or poor emotional resilience. ${ }^{30}$

Health systems can support adults by providing universal healthcare and mental health services, screening services, ${ }^{4}$ brief interventions targeting NCD risk factors in primary care, ${ }^{4}$ and access to affordable drugs for the prevention and control of NCDs. ${ }^{4}$

\section{Older people}

The transition from working adulthood into retirement presents unique opportunities for promoting health as people find new ways to spend their time and resources, while also facing changing identities and relationships. ${ }^{36}$ It is important that as people leave the workplace, they continue to have access to support from other settings, including community centres, primary healthcare programmes, assisted living facilities, hospitals, and home care services. Measures should be taken to maintain functional capacity, strength, and balance of older people ${ }^{8}$ and promote nutrition for older people with diet related NCDs and micronutrient deficiencies. ${ }^{6}$

Mental health must also be targetedfor example, through policies ensuring social support at a time when people often experience social isolation, bereavement, discrimination, financial stress. ${ }^{30}$ This support is often provided at the local level and in cooperation with volunteering activities. Communities must provide appropriate environments for physical activity among older people such as safe neighbourhoods, infrastructure for walking and cycling, and access to recreation facilities, ${ }^{37}$ as well as involving older people in wider social physical activities.

Many surveillance schemes for NCDs exclude older people, and it is worth finding ways to include people older than 69, particularly in the face of ageing populations. Having access to high quality data on NCDs and their risk factors among older people could provide insights for evaluating trends, targeting health promotion interventions, and monitoring the effectiveness of policies aimed to improve the health of older people.

Health systems can ensure promotion of physical activity and healthy nutrition in healthcare settings and residential homes and promote physical activity and nutrition by improving the quality of advice that health professionals give to older people 8

\section{Conclusions}

A life course approach is an underused way to approach NCD prevention and control. Unlike a disease oriented approach, which focuses on interventions for a single condition, a life course approach considers the critical stages, transitions, and settings where large differences can be made in promoting or restoring health. Importantly, it takes into account the social determinants of health, gender, equity, and human rights. It has been emphasised in numerous frameworks and initiatives in the past decade, but more work is needed to give the approach more prominence. Ensuring that the life course perspective is integrated more fully into our work will help us identify appropriate settings for health promotion, design more effective interventions, and ultimately, save lives.

Taking a life course approach requires that we improve health literacy through multisectoral work with individuals, institutions, communities, and countries. Interventions must extend beyond the health sector and be targeted within the natural settings that people encounter through the various stages of their lives. Taking this life course approach will help us to achieve SDG 3.4 and reduce premature mortality by 30\% before 2030 .

Competing interests: We have read and understood BMJ policy on declaration of interests and have no relevant interests to declare. The authors alone are responsible for the views expressed in this article and they do not necessarily represent the decisions, policy, or views of the World Health Organization.

Provenance and peer review: Commissioned; externally peer reviewed.

This article is part of a series proposed by the WHO Global Coordination Mechanism on NCDs and commissioned by The BMJ, which peer reviewed, edited, and made the decision to publish. Open access fees are funded by the Swiss Agency for Development and Cooperation, International Federation of Pharmaceutical Manufacturers and Associations (IFPMA), UNOPS Defeat-NCD Partnership, Government of the Russian Federation, and WHO. For other articles in the series see www.bmj.com/ NCD-solutions

Bente Mikkelsen, director ${ }^{1}$

Julianne Williams, technical officer ${ }^{2}$ Ivo Rakovac, programme manager ${ }^{2}$ Kremlin Wickramasinghe, technical officer ${ }^{2}$ Anselm Hennis, director ${ }^{3}$

Hai-Rim Shin, director ${ }^{4}$

Mychelle Farmer, director ${ }^{5}$

Martin Weber, programme manager ${ }^{1}$

Nino Berdzuli, programme manager ${ }^{1}$

Carina Borges, programme manager ${ }^{2}$

Manfred Huber, coordinator ${ }^{2}$

João Breda, head of office ${ }^{2}$

${ }^{1}$ Division of Non-communicable Diseases and Promoting Health through the Life-course, WHO Regional Office for Europe, Copenhagen, Denmark

${ }^{2}$ WHO European Office for Prevention and Control of NCDs, Moscow, Russia

${ }^{3}$ Department of Noncommunicable Diseases and Mental Health, WHO Pan American Health Organization (PAHO), Washington, DC, USA

${ }^{4}$ Division of Noncommunicable Diseases and Health through the Life-course, WHO Western Pacific Region (WPRO), Manila, Philippines

${ }^{5}$ NCD Child Governing Council, American Academy of Pediatrics/Society for Adolescent Health and Medicine, Itasca, USA

Correspondence to: B Mikkelsen

mikkelsenb@who.int

\section{@ $₫ \Theta$ OPEN ACCESS}

This is an Open Access article distributed under the terms of the Creative Commons Attribution IGO License (https://creativecommons.org/licenses/ by/3.0/igo/), which permits use, distribution, and reproduction in any medium, provided the original work is properly cited.

$$
\text { Check for updates }
$$

1 World Health Organization. The Minsk Declaration: the life-course approach in the context of health 2020. WHO, 2015.

2 Lynch J, Smith GD. A life course approach to chronic disease epidemiology. Annu Rev Public Health 2005;26:1-35. doi:10.1146/annurev. publhealth.26.021304.144505

3 Imura H. Life course health care and preemptive approach to non-communicable diseases. Proc Jpn Acad Ser B Phys Biol Sci 2013;89:462-73. doi:10.2183/pjab.89.462

4 World Health Organization. Tackling NCDs: Best buys and other recommended interventions for the prevention and control of noncommunicable 
diseases. WHO, 2017. https://www.who.int/ncds/ management/best-buys/en/

5 World Health Organization. European action plan to reduce the harmful use of alcohol 2012-2020. WHO, 2012;

6 World Health Organization. European food and nutrition action plan 2015-2020. WHO Europe, 2014.

7 World Health Organization. Good maternal nutrition the best start in life. WHO, 2016. http://www.euro. who.int/_data/assets/pdf_file/0008/313667/ Good-maternal-nutrition-The-best-start-in-life. pdf?ua=1

8 World Health Organization. Physical activity strategy for the WHO European region 2016-2025. WHO Europe, 2016.

9 World Health Organization. Prevention and control of noncommunicable diseases: guidelines for primary health care in low resource settings. WHO, 2012.

10 World Health Organization. Noncommunicable Diseases: Key facts. 2018. https://www.who.int/ news-room/fact-sheets/detail/noncommunicablediseases

11 World Health Organization. Global Action Plan for the Prevention and Control of NCDs 2013-2020. https:// www.who.int/nmh/events/ncd_action_plan/en/

12 Barker DIP. Mothers, babies and health in later life. Elsevier Health Sciences, 1998.

$13 \mathrm{Ku} \mathrm{GMV}$. Life-course approach in the control and prevention of chronic diseases. Available from, 2018 https://www.bmj.com/rapid-response/2011/11/03/ life-course-approach-control-and-prevention-chronicdiseases

14 Rutter H, Savona N, Glonti K, et al. The need for a complex systems model of evidence for public health. Lancet 2017;390:2602-4. doi:10.1016/ S0140-6736(17)31267-9

15 WHO. Report of the commission on ending childhood obesity. WHO, 2016

16 World Health Organization. Shanghai declaration on promoting health in the 2030 agenda for sustainable development. WHO, 2016

17 World Health Organization. Vienna declaration on nutrition and noncommunicable diseases in the context of health 2020. 2013. http://www.euro. who.int/ data/assets/pdf file/0005/193253/ CONSENSUS-Vienna-Declaration-5-July-2013.pdf

18 World Health Organization. World report on ageing and health. World Health Organization, 2015.

19 World Health Organization. Health 2020: the European policy for health and wellbeing. http:// www.euro.who.int/en/health-topics/health-policy/ health-2020-the-european-policy-for-health-andwell-being

20 World Health Organization. Action plan for the prevention and control of noncommunicable diseases in the WHO European Region. WHO Europe, 2016.

21 World Health Organization. Ambient air pollution: health impacts https://www.who.int/airpollution/ ambient/health-impacts/en/

22 Rutter H, Savona N, Glonti K, et al. The need for a complex systems model of evidence for public health. Lancet 2017;390:2602-4. doi:10.1016/ S0140-6736(17)31267-9

23 World Health Organization. Tobacco-free generations: protecting children from tobacco in the WHO European Region. WHO Europe, 2017.

24 World Health Organization. Good maternal nutrition the best start in life. 2016. http://www.euro.who. int/en/health-topics/disease-prevention/nutrition/ publications/2016/good-maternal-nutrition.-thebest-start-in-life-2016.

25 Hutin Y, Desai S, Bulterys M. Preventing hepatitis B virus infection: milestones and targets. Bulletin of the World Health Organization 2018;96:443-A.

26 Davison KK, Lawson CT. Do attributes in the physical environment influence children's physical activity? A review of the literature. Int I Behav Nutr Phys Act 2006:3:19 doi:10.1186/1479-5868-3-19

27 World Health Organization. WHO European Childhood Obesity Surveillance. http://www.euro. who.int/en/health-topics/disease-prevention/ nutrition/activities/who-european-childhoodobesity-surveillance-initiative-cosi

28 Pechmann C, Levine L, Loughlin S, Leslie F. Impulsive and self-conscious: Adolescents vulnerability to advertising and promotion. J Public Policy Mark 2005;24:202-21. doi:10.1509/ jppm.2005.24.2.202

29 Patel V, Flisher AJ, Hetrick S, McGorry P. Mental health of young people: a global public-health challenge.
Lancet 2007:369:1302-13. doi:10.1016/S01406736(07)60368-7

30 World Health Organization (EURO). The health and well-being of men in the WHO European Region: better health through a gender approach. http://www.euro.who.int/en/health-topics/ health-determinants/gender/publications/2018/ the-health-and-well-being-of-men-in-the-whoeuropean-region-better-health-through-a-genderapproach-2018

31 Inchley J. Adolescent obesity and related behaviours: trends and inequalities in the WHO European region, 2002-2014: Observations from the Health Behavior in School-aged Children (HBSC) WHO Collaborative Cross-national Study. WHO Europe, 2017.

32 WHO. Investing in children: The European child and adolescent health strategy 2015-2020. WHO Europe, 2014.

33 Warburton DER, Bredin SSD. Health benefits of physical activity: a systematic review of current systematic reviews. Curr Opin Cardiol 2017;32:54156. doi:10.1097/HCO.0000000000000437

34 Marten R, Kadandale S, Butler J, et al, Sugar, Tobacco, and Alcohol Taxes (STAX) Group. Sugar, tobacco, and alcohol taxes to achieve the SDGs. Lancet 2018;391:2400-1. doi:10.1016/S0140 6736(18)31219-4

35 Thow AM, Downs SM, Mayes C, Trevena H, Waqanivalu T, Cawley J. Fiscal policy to improve diets and prevent noncommunicable diseases: from recommendations to action. Bull World Health Organ 2018;96:201-10. doi:10.2471/ BLT.17.195982

36 National Institute on Aging. The health \& retirement study. National Institutes of Health, US Department of Health \& Human Resources. 2012. http://hrsonline. isr.umich.edu/.

37 World Health Organization. Age-friendly environments in Europe: A handbook of domains for policy action. WHO, 2017

Web appendix 1: Thinking across the life course

Cite this as: $B M / 2019 ; 364: 1257$

http://dx.doi.org/10.1136/bmj.1257 